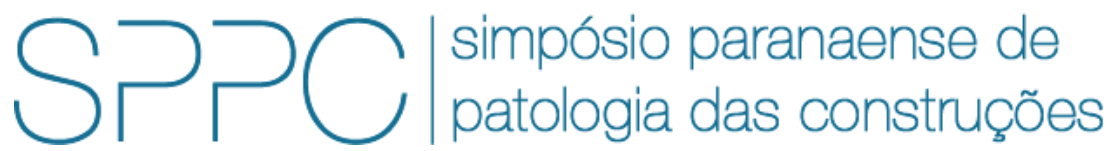

ISSN 2526-7248 artigo 2SPPC1008, pp. 81-88, 2017

\title{
Desenvolvimento de dispositivo rotacional em plasma CC pulsado para a deposição de filmes finos de nanomateriais em agregados graúdos sujeitos à reação álcali-agregado
}

\author{
MAZUR, M. M. ${ }^{1,3}$; PORTELLA, K. F. ${ }^{1 *}$; BRAGANÇA, M. O. G. P. ${ }^{1}$; SILVA, E. de M. ${ }^{1} ;$ PIANARO, S. \\ A. ${ }^{2} ;$ RIBEIRO, B. E. ${ }^{1}$; ALBERTI, E4 . \\ 1 Institutos Lactec, portella@lactec.org.br; mzmauricio@hotmail.com; mariana.portella@lactec.org.br; \\ evandro.silva@lactec.org.br; bruno.ribeiro@lactec.org.br \\ $1^{*}$ Bolsista do CNPq, Brasil, processo 302672/2016-8 \\ 2Universidade Estadual de Ponta Grossa, sap@uepg.br \\ 3Universidade Federal do Paraná \\ ${ }^{4}$ Centrais Elétricas do Rio Jordão, emerson@elejor.com.br
}

\begin{abstract}
Resumo. A reação álcali-agregado (AAR) é um tipo de ataque químico presente em boa parte dos concretos de cimento Portland. São reações químicas formadas normalmente por álcalis, os quais estão presentes no agregado, no cimento, na água de amassamento e nos agentes externos ambientais. O produto da sua interação é um silicato amorfo com característica de um gel higroscópico, cuja expansão propaga trincas na matriz e no agregado, resultando em fenômenos deletérios para estruturas civis. Para reduzir estes efeitos, alguns trabalhos apresentam alternativas como a adição de materiais pozolânicos, compostos a base de lítio, nano- $\mathrm{Fe}_{3} \mathrm{O}_{4}$, nano- $\mathrm{TiO}_{2}$, entre outros. Neste trabalho, teve-se como objetivo desenvolver uma forma de depositar nano-Al sobre a superfície de agregados com a utilização da técnica de plasma magnetron sputtering. Para utilizar esta técnica, foi necessário rotacionar os agregados para que houvesse a formação do filme fino em toda a superfície, uma vez que a deposição acontece no sistema de forma direcional. Neste sentido, desenvolveu-se um dispositivo cilíndrico rotacional para haver uma homogeneidade do filme formado. As análises de microscopia óptica demonstraram haver um recobrimento em uma batelada de $500 \mathrm{~g}$ do agregado, sendo avaliada a sua eficácia a partir dos testes de expansão em meios de envelhecimento acelerado. Os resultados quanto à expansão dos agregados in natura mostraram valores médios de $(0,765 \pm 0,011) \%$, enquanto que os recobertos com os filmes nano de alumínio de $(0,160 \pm 0,003) \%$, aos 30 dias, respectivamente.
\end{abstract}

Palavras-chave: RAA, plasma CC pulsado, agregado de concreto, nano alumínio.

Abstract. The alkali-aggregate (AAR) reaction is a type of chemical attack presented in most concrete Portland cement. They are chemical reactions normally formed by alkalis, which are presented in the aggregate, cement, kneading water and environmental external agents. The product of its interaction is an amorphous silicate with the characteristic of a hygroscopic gel, whose expansion propagates cracks in the matrix and in the aggregate, resulting in deleterious phenomena for civil structures. To reduce these effects, there are studies with alternatives such as the addition of pozzolanic materials, lithium-based compounds, nano- $\mathrm{Fe}_{3} \mathrm{O}_{4}$, nano- $\mathrm{TiO}_{2}$, among others. In this work, was aimed to develop a way of depositing nano-Al on the surface of aggregates using the magnetron sputtering plasma technique. In order to use this technique, it was necessary to rotate the aggregates so that the thin film formed on the whole surface, since the deposition happens in the system in a directional way. For that, a rotational cylindrical device was developed to have homogeneity of the formed film. Optical microscopy analysis showed that the coating was done in a batch of $500 \mathrm{~g}$ of the aggregate, and its effectiveness was evaluated from the expansion tests in accelerated aging media. The results on the expansion of the reference aggregates (without film) showed average values of $(0.765 \pm 0.011) \%$, whereas those covered with nano aluminum films of $(0.160 \pm 0.003) \%$, at 30 days, respectively.

Keywords: AAR, plasma DC pulsed, concrete aggregates, nano Al. 
MAZUR, M. M.; ET AL., DESENVOLVIMENTO DE DISPOSITIVO ROTACIONAL EM PLASMA CC PULSADO PARA A DEPOSIÇÃO DE FILMES FINOS DE NANOMATERIAIS EM AGREGADOS GRAÚDOS SUJEITOS À REAÇÃO ÁLCALI-AGREGADO. $2^{\circ}$ Simpósio Paranaense de Patologia das Construções (20 SPPC), artigo 2SPPC1008, pp. 81-88, 2017. DOI: 10.4322/2SPPC.2017.008

\section{Introdução}

As reações alcali agregado (AAR) ocorrem devido à presença de hidróxidos alcalinos constituintes no concreto de cimento Portland. Essas reações produzem um silicato amorfo com característica de gel, o qual, devido a sua expansão propaga trincas na matriz e no agregado, sendo uma das manifestações patológicas mais deletérias nestes tipos de estruturas [1]. Com intuito de reduzir a AAR, a utilização de cimentos com baixo teor de alcalis, agregados inertes, a adição de minerais pozolânicos e a diminuição da umidade local, tornam-se alternativas viáveis, no entanto, o controle dessas variáveis gera custos relativamentes altos [2]. A adição de $15 \%$, em massa, de cinzas volantes foi descrita por Lucca [3], no controle da AAR em cimentos Portland.

Estudos de adição de nano- $\mathrm{Fe}_{3} \mathrm{O}_{4}$ em cimento Portland em idades iniciais demonstraram haver certa estabilidade eletroquímica em seus concretos, segundo Bragança [4], inferindo-se, adicionalmente, que com $1 \%$ nano- $\mathrm{Fe}_{3} \mathrm{O}_{4}$, em massa, conseguiu-se, também, uma redução dos índices de absorção de água e de vazios, conferindo ao concreto menor susceptibilidade à corrosão.

Sanchez [5], comentou a necessidade do desenvolvimento e da aplicação de nano tecnologia na alteração das características elétricas, mecânicas e térmicas em concretos, buscando mitigar a degradação de estruturas civil, sendo esta, parte integrante deste trabalho de pesquisa.

O plasma é um dos estados da matéria e estima-se que mais de $99 \%$ do universo seja constituído por ele, estando presente na natureza em diversos fenômenos como raios, aurora boreal e, no cosmos, nas estrelas. Em nossas residências, o plasma está presente nas lâmpadas fluorescentes, televisão de plasma, na chama de uma vela e em motores a combustão.

Plasmas tecnológicos utilizados em indústrias e em laboratórios são importantes devido a alta potência e à densidade de energia. Dentre os benefícios do processo a plasma, pode-se destacar o baixo consumo energético, a redução ou a extinção de gases tóxicos e ou sub-produtos, a redução de matéria prima em tratamentos superficiais e a redução do preço do produto final [6].

Plasmas tecnológicos são gerados das mais diversificadas fontes, como por exemplo: microondas, rádio frequência $(R F)$, cátodo oco, vaporização por arco, corrente contínua, corrente alternada, incluíndo a utilização do magnetron sputtering e das possibilidades de combinação destes. Nesses casos para a geração do plasma há a necessidade de que os elétrons recebam energia suficiente para gerar colisões nos átomos e moléculas e ionizá-los [7-8]. Das reações que ocorrem no plasma, as mais importantes são: ionização $\left(\mathrm{e}^{-}+\mathrm{X} \rightarrow \mathrm{X}_{+}+\mathrm{e}^{-}\right)$, dissociação molecular ( $\left.\mathrm{e}^{-}+\mathrm{XY} \rightarrow \mathrm{X}+\mathrm{Y}+\mathrm{e}^{-}\right)$, excitação $\left(\mathrm{e}^{-}+\mathrm{X} \rightarrow \mathrm{X}^{*}+\mathrm{e}^{-}\right)$e recombinação $\left(\mathrm{X}^{*}+\right.$ $\mathrm{e}^{-} \rightarrow \mathrm{X}$ ) [7]. Nos casos de sistemas de RF, os elétrons do próprio gás são retirados de sua órbita e o gás torna-se ionizado. Esses processos fornecem íons provenientes de gases nobres $(\mathrm{Ar}, \mathrm{He})$ ou reativos $\left(\mathrm{O}_{2}\right)$, gases precursores $\left(\mathrm{SF}_{6}\right)$, decomposição molecular e de metais condensáveis para a formação de filmes, corrosão superficial ou apenas limpeza de superfícies [8].

Sistemas de magnetron sputtering são amplamente utilizados devido a possibilidade 
MAZUR, M. M.; ET AL., DESENVOLVIMENTO DE DISPOSITIVO ROTACIONAL EM PLASMA CC PULSADO PARA A DEPOSIÇÃO DE FILMES FINOS DE NANOMATERIAIS EM AGREGADOS GRAÚDOS SUJEITOS À REAÇÃO ÁLCALI-AGREGADO. $2^{\circ}$ Simpósio

Paranaense de Patologia das Construções (20 SPPC), artigo 2SPPC1008, pp. 81-88, 2017. DOI: 10.4322/2SPPC.2017.008

de formação de filmes em grandes áreas. Os íons gerados são confinados e acelerados em um campo magnético fornecido por imãs permanentes. Os íons do gás de trabalho são acelerados contra um alvo, normalmente do material a ser depositado. A aceleração desses íons contra o alvo gera o desarranjo e ejeção do material e consequente deposição em todo o reator [8].

Neste trabalho, foi proposto, como objetivo principal, a aplicação e a adaptação da técnica de plasma magnetron sputtering (plasma de corrente contínua pulsada) em agregados utilizados na construção civil, no sentido de recobri-los com filmes nanométricos de alumínio, e de se promover a mitigação da RAA em concretos de cimento Portland.

\section{Materiais e métodos}

\subsection{Reator de plasma}

O sistema de plasma foi confeccionado em vidro PIREX e em alumínio aeronáutico com um volume total do reator de $28 \mathrm{~L}$, conforme ilustrado na Figura 1. A abertura do reator foi fabricada de forma longitudinal, sendo fechado herméticamente com vedações em VITON. O vácuo foi gerado por duas bombas, uma mecânica para um pré vácuo e uma bomba difusora, marca EDWARDS, para a obtenção de pressões finais no sistema, abaixo de $10^{-5} \mathrm{mbar}$. $O$ gás de trabalho utilizado foi o argônio, devido a ser inerte no processo e a sua vazão controlada por sistema, marca, Emiatec SFM5000. A fonte de tensão utilizada foi do tipo de corrente contínua pulsada [9] A tensão de trabalho foi de $800 \mathrm{Vp}$ com 1,3 Ap de corrente e ciclo de pulsos de $50 \%$. Os alvos de Al foram construídos nas dimensões de $(80 \times 245) \mathrm{mm}$ e foram colocados em um sistema magnético do tipo "balance". Após gerar o vácuo até $10^{-5}$ mbar, a pressão de trabalho foi estabilizada com o gás argônio a uma pressão de $10^{-3}$ mbar, à qual foi a pressão ideal para a deposição desses elementos.

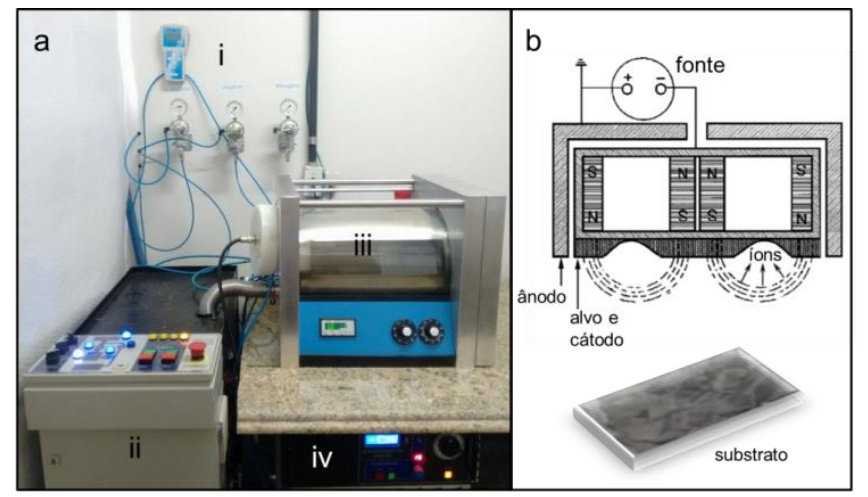

Figura 1: (a) Sistema de plasma CC pulsado trabalhado, sendo composto de: i) entrada de gases; ii) paínel de controle; iii) o reator propriamente dito; e (iv) a fonte de tensão (iv); e em b) vista esquemática da seção transversal do magnetron. Adaptado [8]. 
MAZUR, M. M.; ET AL., DESENVOLVIMENTO DE DISPOSITIVO ROTACIONAL EM PLASMA CC PULSADO PARA A DEPOSIÇÃO DE FILMES FINOS DE NANOMATERIAIS EM AGREGADOS GRAÚDOS SUJEITOS À REAÇÃO ÁLCALI-AGREGADO. $2^{\circ}$ Simpósio

Paranaense de Patologia das Construções (20 SPPC), artigo 2SPPC1008, pp. 81-88, 2017. DOI: 10.4322/2SPPC.2017.008

\subsection{Agregados}

Agregados provenientes de uma jazida basáltica da região sul do Brasil, no município de Candói, Paraná, foram utilizados nos testes. O agregado foi britado com diâmetro médio de $9,5 \mathrm{~mm}$. Os testes de reatividade seguiram a norma NBR 15577 [10].

Foi realizada microscopia óptica, utilizando um microscópio BX51-Olympus para avaliar a deposição direcional dos filmes de alumínio. Para o emprego desta técnica, o agregado utilizado foi cortado com um disco de diamante de dimensões aproximadas de $(125 \times 0,375 \times 12,5) \mathrm{mm}$, no sentido de obter uma região plana superficial.

Os agregados foram lavados previamente em água corrente, colocados em formas e permaneceram em estufa de secagem a $65^{\circ} \mathrm{C}$ até serem utilizados no reator. Esse procedimento garantiu a redução de contaminantes, como partículas de poeira, auxiliando na geração de vácuo.

\subsection{Desenvolvimento do dispositivo para a acomodação dos agregados no reator de plasma}

O desenvolvimento do dispositivo de acomodação e rolagem dos agregados no interior do plasma, dentro do reator, foi iniciado com o projeto de um jarro cilíndrico para comportar as britas. Foi utilizado um cilindro polimérico de $(29,5 \times 22,0) \mathrm{mm}$, conforme ilustrado na Figura 2 (a), que posteriormente foi cortado nas laterais (Figura 2 (b)) e revestidos com uma tela em alumínio com $5,5 \mathrm{~mm}$ de malha (Figura 2 (c)) para dar suporte a uma tela com malha $0,5 \mathrm{~mm}$. Dessa forma, como projetado, o jarro polimérico isolou o cátodo do ânodo não havendo geração de descargas elétricas indesejadas durante a deposição.

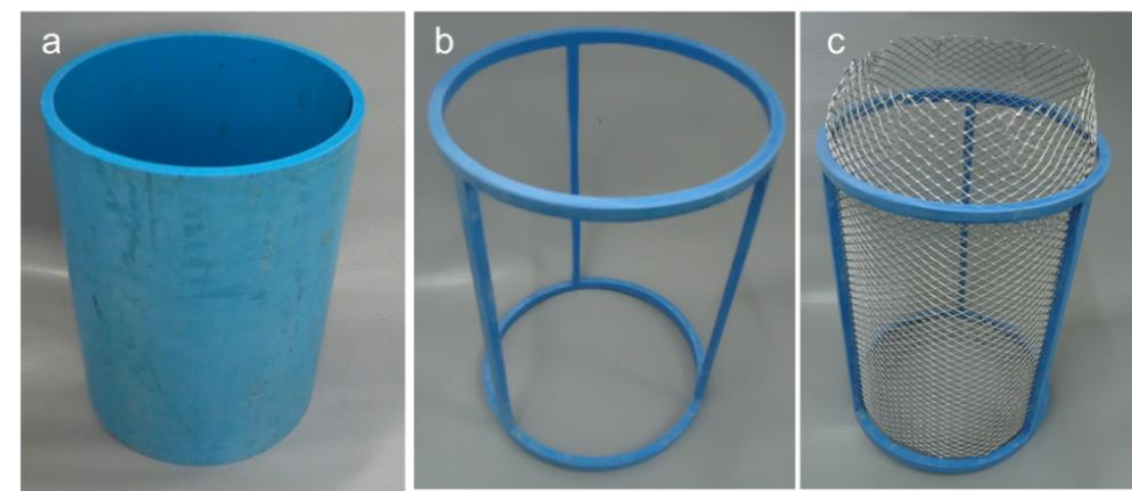

Figura 2: (a) Cilindro polimérico utilizado para a confecção do jarro rotacional; (b) corte das laterais do jarro; e (c) jarro montado com a tela de alumínio para a deposição.

Para a rotação do jarro foi desenvolvido um sistema de rolos (Figura 3 (a) (i)), em alumínio e, também, um eixo com vedações de O'rings, em Viton, com polias e correia dentada Figura 3 (a) (ii). O sistema foi fixado na porta do reator, conforme está ilustrado na Figura 3 (a). Ele foi construído de forma a suportar o peso da brita durante a deposição. Testes iniciais demonstraram que este não foi um limitante e sim o volume de material, o qual ficou definido em $500 \mathrm{~g}$. Na Figura 3 (b) é possível visualizar o sistema fechado com o jarro e sistema rotacional. $O$ tracejado em 
MAZUR, M. M.; ET AL., DESENVOLVIMENTO DE DISPOSITIVO ROTACIONAL EM PLASMA CC PULSADO PARA A DEPOSIÇÃO DE FILMES FINOS DE NANOMATERIAIS EM AGREGADOS GRAÚDOS SUJEITOS À REAÇÃO ÁLCALI-AGREGADO. $2^{\circ}$ Simpósio

Paranaense de Patologia das Construções (20 SPPC), artigo 2SPPC1008, pp. 81-88, 2017. DOI: 10.4322/2SPPC.2017.008

vermelho indica a posição do magnetron (Figura 3 (b) (iii)) e, em amarelo, o posicionamento do agregado (Figura 3 (b) (iv)).

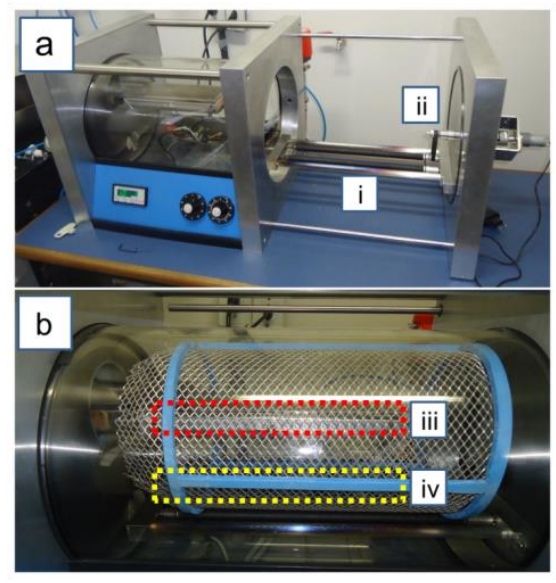

Figura 3: Vista geral do reator de plasma CC pulsado: (a) com o sistema rotacional fixado na porta do reator; e (b) sistema fechado.

\section{Resultados e Discussões}

Na Figura 4 (a), é possível visualizar de forma geral a alocação do dispositivo suporte e do agregado dentro do reator. Como visto, o dispositivo rotacional projetado ficou perfeitamente alocado entre o magnetron e o alvo a ser depositado, propiciando uma melhor eficiência na deposição dos filmes finos. Na Figura 4 (b) é possível visualizar em tonalidade diferenciada como os agregados ficaram posicionados no interior do plasma formado.

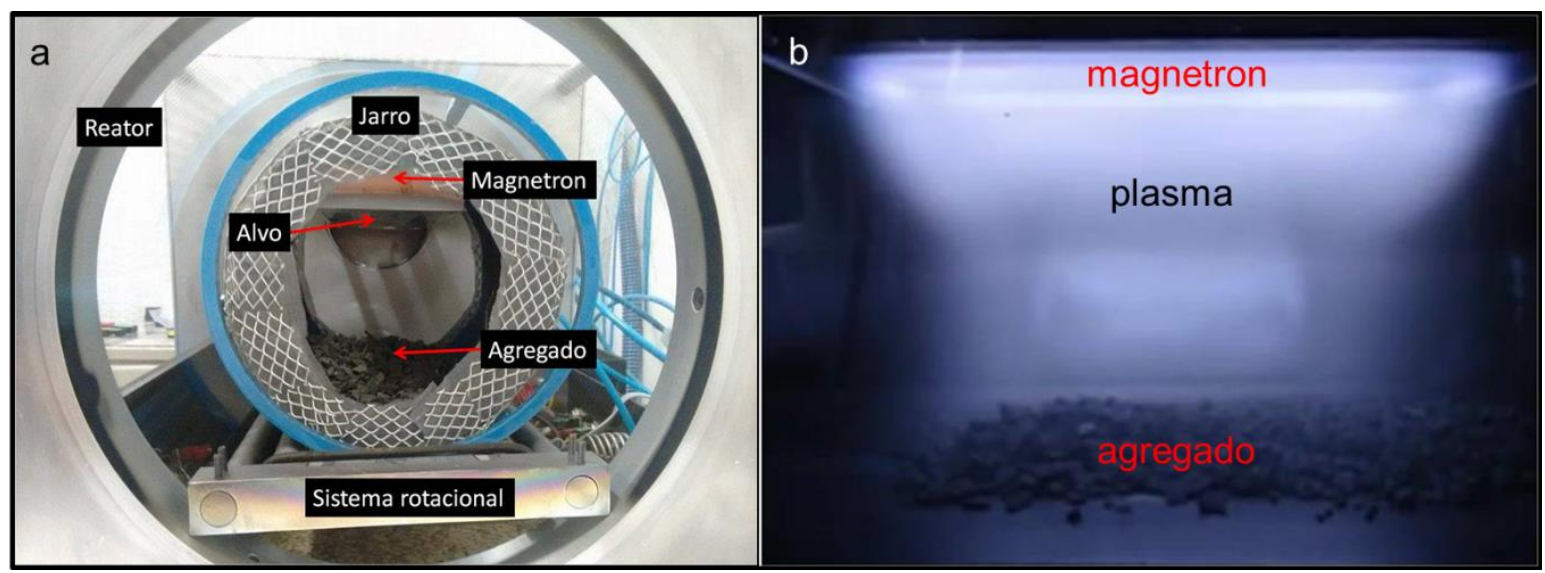

Figura 4: (a) Vista interna do reator de plasma CC pulsado, contendo o cilindro projetado para a acomodação de uma batelada de $500 \mathrm{~g}$ de agregado, e (b) vista parcial do interior do reator com o plasma de argônio sobre a batelada de $500 \mathrm{~g}$ de agregado.

Testes prévios demonstraram que a rotação ideal para o tombamento dos agregados foi de $2 \mathrm{rpm}$. Houve dificuldade na obtenção de vácuo devido as características de porosidade do substrato. Os poros fechados formam vazamentos virtuais, os quais demandam tempo de trabalho do equipamento. Em média, uma 
MAZUR, M. M.; ET AL., DESENVOLVIMENTO DE DISPOSITIVO ROTACIONAL EM PLASMA CC PULSADO PARA A DEPOSIÇÃO DE FILMES FINOS DE NANOMATERIAIS EM AGREGADOS GRAÚDOS SUJEITOS À REAÇÃO ÁLCALI-AGREGADO. $2^{\circ}$ Simpósio Paranaense de Patologia das Construções (20 SPPC), artigo 2SPPC1008, pp. 81-88, 2017. DOI: 10.4322/2SPPC.2017.008

batelada de $500 \mathrm{~g}$ de agregado demanda neste tipo de reator de bancada, previamente ao depósito, 24 h de preparo do vácuo.

Filmes com tempos superiores e de forma direcional, ou seja, sem o sistema rotacional, foram depositados com o intuito de verificar a sua morfologia na superfície do agregado. Na Figura 5 (a) é possível visualizar a interface agregado in natura e o filme de alumínio (destaque da região com a tarja de $500 \mu \mathrm{m}$ ), respectivamente, por mudanças na tonalidade original da superfície do agregado.

Os resultados experimentais das deposições efetuadas nas condições trabalhadas indicaram a deposição de filmes de alumínio na ordem de $1 \mathrm{~nm} \cdot \mathrm{s}^{-1}$. Em condições rotacionais estimou-se que os filmes ou cluster formados possuíram em média 20 $\mathrm{nm}[9]$.

Os resultados de reatividade dos agregados tratados a plasma foram apresentados por Portella [11]. Os testes de expansão transcorreram por 30 dias, em acordo com a norma [10], com um limite máximo de expansão de $0,19 \%$. A análise dos resultados dos testes executados com o agregado limpo natural, ou seja, sem a deposição (Figura 6 (a)), mostrou uma reação de expansão acima do valor recomendado em norma no quarto dia de teste, com uma expansão média de $(0,765 \pm 0,011) \%$, ou seja, cinco vezes aos valores apresentados com os filmes revestidos. O filme de alumínio (Figura 6 (b)) apresentou, respectivamente $(0,160 \pm$ $0,003) \%$.

Novas metodologias para a aspersão dos filmes estão sendo desenvolvidas para que se consiga uma redução nos custos energéticos e de produção em escala industrial. A substituição da fonte de geração de plasma por uma que opere em condições de temperatura e pressão normais elimina o sistema de vácuo e reduz o tempo ocioso na sua geração, causado, principalmente, nesta etapa mais crítica do processo.

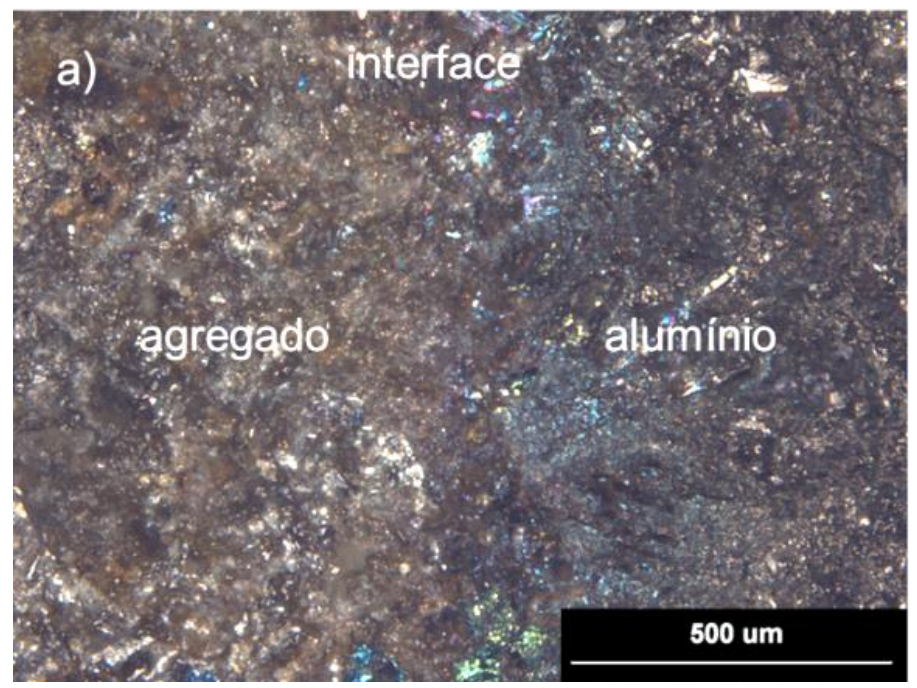

Figura 5: Imagem por microscopia óptica do filme de alumínio depositado de forma direcional sobre a superfície de agregados, com tempo de 10 minutos, em destaque, este foi marcado com a tarja da ordem de $500 \mu \mathrm{m}$. 
MAZUR, M. M.; ET AL., DESENVOLVIMENTO DE DISPOSITIVO ROTACIONAL EM PLASMA CC PULSADO PARA A DEPOSIÇÃO DE FILMES FINOS DE NANOMATERIAIS EM AGREGADOS GRAÚDOS SUJEITOS À REAÇÃO ÁLCALI-AGREGADO. $2^{\circ}$ Simpósio Paranaense de Patologia das Construções (20 SPPC), artigo 2SPPC1008, pp. 81-88, 2017. DOI: 10.4322/2SPPC.2017.008

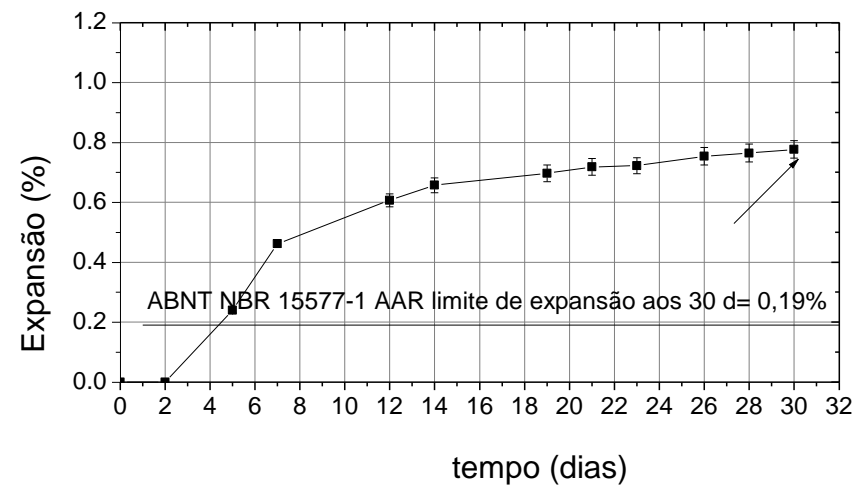

(a)

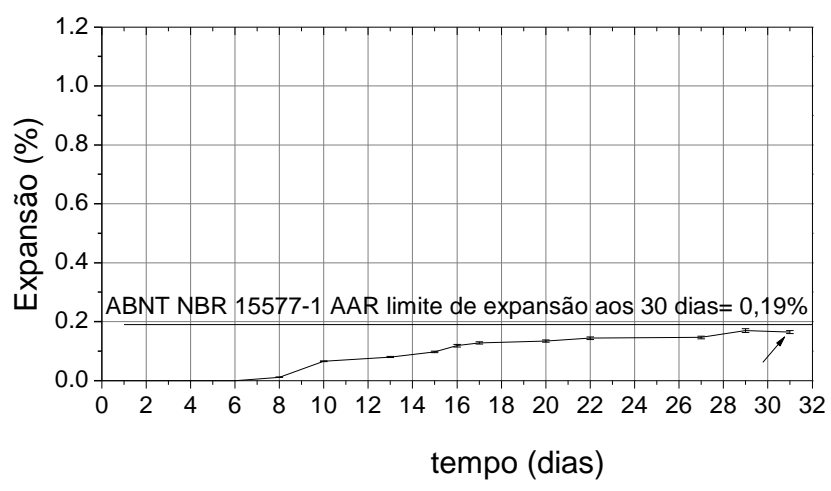

(b)

Figura 6: Resultados dos testes de expansão (\%), em função do tempo de exposição em solução alcalina, em dias: (a) do agregado in natura; e (b), com revestimento em nano alumínio, segundo a NBR 15577, parte 5 [10].

\section{Conclusão}

A técnica de plasma magnetron spputering demonstrou que se pode obter resultados eficientes na mitigação da RAA com a deposição de nano materiais metálicos, principalmente, por Al, sobre a superfície dos agregados.

As alterações desenvolvidas no reator a plasma foram compatíveis com os substratos utilizados, demonstrando que a técnica foi promissora para a sua aplicação na construção civil.

\section{Agradecimentos}

Os autores agradecem à infraestrutura e apoios em recursos humanos e financeiros à ELEJOR, projeto P\&D 2945-0004/2013; à ANEEL; à COELBA, projeto P\&D 00470047/2011; à ANEEL; ao CNPq, Lei 8010/90, principalmente à LI 14/3445028-1 DI_14/2190047-7; ao CNPq bolsas PIBIT e DT, processo 302672/2016-8; aos Institutos Lactec; à Universidade Federal do Paraná/PIPE; e à Universidade Estadual de Ponta Grossa/DEMA - Departamento de Engenharia de Materiais e LIMAC. 
MAZUR, M. M.; ET AL., DESENVOLVIMENTO DE DISPOSITIVO ROTACIONAL EM PLASMA CC PULSADO PARA A DEPOSIÇÃO DE FILMES FINOS DE NANOMATERIAIS EM AGREGADOS GRAÚDOS SUJEITOS À REAÇÃO ÁLCALI-AGREGADO. $2^{\circ}$ Simpósio Paranaense de Patologia das Construções (20 SPPC), artigo 2SPPC1008, pp. 81-88, 2017. DOI: 10.4322/2SPPC.2017.008

\section{Referências}

[1] Tambelli, C.E.; Schneider, J.F.; Hasparyk, N.P.; Monteiro, P.J.M. (2006) Study of the structure of alkali-silica reaction gel by high-resolution NMR spectroscopy. Journal of Non-Crystalline Solids, 352: 3429-3436.

[2] Hasparyk, N.P. (1999). Investigação dos mecanismos da reação álcaliagregado - efeito da cinza da casca de arroz e da sílica ativa. Dissertação (Mestrado), Universidade Federal de Goiás, Escola de Engenharia Civil, Goiânia. 257.

[3] Lucca, A. C. K. (2010) Reação álcali-agregado: efeito do uso de cinzas volantes. Trabalho de conclusão de curso, Escola de Engenharia da Universidade Federal do Rio Grande do Sul. Porto Alegre, Brasil.

[4] Bragança, M.O.G.P.; Portella, K.F.; Bonato, M.M.; Alberti, E.; Zarbin, C.E.M. (2016) Performance of Portland cement concretes with 1\% nano-Fe3O4 addition: Electrochemical stability under chloride and sulfate environments. Construction \& Building Materials, 117:152-162.

[5] Sanchez, F.; Sobolev, K. (2010). Nanotechnology in concrete - a review. Construction and Building Materials, 24:2060-2071.

[6] Roth, J. R. Industrial Plasma Engineering (2001). Institute of Physics Publishing Bristol and Philadelphia.

[7] Lieberman, M.; Lichtenberg, A. (1994). Principles of Plasma Discharge and Materials Processing. John Wiley \& Sons.

[8] Anders, A. (2005) Plasma and ion sources in large area coatings: A review, Surface and coatings technology, 200:1893-1906.

[9] Mazur, M.M. (2014) Desenvolvimento de superfícies funcionais com filmes de titânio para isoladores elétricos cerâmicos utilizando a técnica de pulsado CC plasma magnetron sputtering. Tese de doutorado, UFPR/PIPE (em andamento).

[10] ABNT (2008). NBR 15577, partes-1-3-4-5: 2008. Agregados - Reatividade álcali-agregado, Partes 1, 3, 4 e 5: Guia para avaliação da reatividade potencial e medidas preventivas para uso de agregados em concreto. Brasil.

[11] Portella, K. F; Mazur, M. M; Bragança, M. O. G. P; Silva, E. De M; Bronholo, J. L; Pianaro, S. A; Alberti, E. L. (2016) Plasma CC pulsed as a tool for generating mitigation nanomaterials alkaliaggregate reaction in Portland cement concrete, XII International Conference on Structural Repair and Rehabilitation, 26-29. 